\title{
Catadioptric Optics for Laser Doppler Velocimeter Applications
}

\section{Stephen E. Dunagan}




\section{Catadioptric Optics for Laser Doppler Velocimeter Applications}

Stephen E. Dunagan, Ames Research Center, Moffett Field, California 


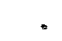

. 


\title{
Catadioptric Optics for Laser Doppler Velocimeter Applications
}

\author{
Stephen E. Dunagan \\ Ames Research Center
}

SUMMARY

In the design of a laser velocimeter system, attention must be given to the performance of the optical elements in their two principal tasks: focusing laser radiation into the probe volume, and collecting the scattered light. For largeaperture applications, custom lens design and fabrication costs, long optical path requirements, and chromatic aberration (for two-color operation) can be problematic. The adaptation of low-cost Schmidt-Cassegrain astronomical telescopes to perform these laser-beam manipulation and scattered-light collection tasks is examined. A generic telescope design is analyzed using ray-tracing and Gaussian-beam-propagation theory, and a simple modification procedure for converting from infinite to near-unity conjugate-ratio operation with image quality near the diffraction limit has been identified. Modification requirements and performance are predicted for a range of geometries. Finally, a 200-mm-aperture telescope was modified for $\mathrm{f} / 10$ operation; performance data for this modified optic for both laser-beam focusing and scattered-light collection tasks agree well with predictions.

\section{INTRODUCTION}

The dual-beam laser Doppler velocimeter (LV) has become the instrument of choice for nonintrusive measurement of fluid velocity at a point (ref. 1). A variety of commercial LV systems are available, but it is still often necessary to configure a custom system, particularly for long-range (greater than $2-\mathrm{m}$ ) applications. The specification of a suitable optical configuration is critical in determining the cost and performance of the end product.

The optical tasks associated with dual-beam LV are twofold. First, the Gaussian-profile laser output must be divided and the two beams must be focused to an intersection that defines the probe volume. A very stable system is obtained if a parallel output beamsplitter and co-axial focusing optics are used. The required sensitivity (beam angle) and operating range combine to identify a minimum aperture for a dual-beam configuration. Second, the scattered light from the probe 
volume must be collected and directed to a photodetector. The probe volume must be sharply imaged if spatial filtering is to be used to reject unwanted scattered light from optical or model surfaces. Furthermore, for long-range operation, a large aperture is needed to offset the inverse-square decrease in scattered-light intensity. These tasks may be performed by two separate optical systems (off-axis collection) or by the same set of optics (confocal backscatter collection). Zoom features may also be desired in order to easily relocate the probe volume along the optical axis. The required aperture may be as large as several hundred millimeters for long-range systems.

Well-corrected lenses generally perform these functions in commercial and short-range custom LV systems. A broad selection of off-the-shelf lenses is available in apertures up to $75 \mathrm{~mm}$, and some stock lenses are available in apertures to 150 $\mathrm{mm}$. For larger apertures, a custom-designed lens is almost certainly required with the associated high design and manufacturing costs. If multicolor operation is desired, the need for chromatic correction limits the performance of stock optics and complicates the design problem for custom lenses.

In recent years, the growing commercial availability of catadioptric (combination of refractive and reflective elements) telescopes in the 100- to $350-\mathrm{mm}$ aperture range has provided an alternative to lens optics. Owing to marketing competition and advanced manufacturing techniques, the cost of these units is quite low. The popular Cassegrain configuration with its folded path is very compact. The focusing mechanism provided with a telescope is an attractive feature for zoom applications. Furthermore, the low curvature and low thickness of the single refractive element provide very low chromatic aberration.

However, the intended telescope imaging task of astronomical viewing at infinite conjugate ratio (object distance to image distance) is considerably removed from the LV beam focusing and probe-volume imaging tasks at conjugate ratios near unity. The near-focus specification for commercial telescopes is typically about 50 times the primary aperture ( $/ 50)$, whereas the ratio of the probe-volume distance to the collector aperture (f-ratio) for a long-range LV system is closer to 10 . A modification of the telescope is clearly required. The purpose of this study is to examine how one might adapt a Schmidt-Cassegrain telescope for LV operation, and to evaluate both the predicted and experimentally measured performance of a modified unit by comparison with similar lens optics.

\section{SCHMIDT-CASSEGRAIN OPTICS}

The basic elements of a Schmidt-Cassegrain telescope are shown in figure 1. The Cassegrain telescope consists of a concave primary mirror, followed by a convex secondary mirror which focuses light through a central aperture in the primary to the Cassegrain focus. Aspheric primary or secondary surfaces can provide an 
aberration-free system at infinite conjugate. However, aspheric surfaces are very difficult to manufacture. The Schmidt corrector plate, having a slightly figured front (left) surface and a planar back surface, "pre-corrects" the spherical aberration introduced by the spherical primary and secondary mirrors. A fourth-order corrector plate having a figuring depth d given by (ref. 2).

$$
d=A_{2} r^{2}+A_{4} r^{4}
$$

(where $A_{2}$ and $A_{4}$ are the second- and fourth-order-aspheric constants and $r$ is the radius) can be optimized to provide diffraction-limited imaging at infinite conjugate. The corrector plate also supports the secondary mirror.

Possible modifications to the telescope to perform the beam focusing and scattered-light collection tasks of LV operation are shown in figures 2 and 3 . For confocal backscatter operation, the cylinder of backscattered light shown in figure 3 is focused by a lens to the photodetector. A simple thin-lens analysis of the primarysecondary mirror system indicates that mirror spacing or mirror curvature must be modified for either task. For any such modification, the Schmidt corrector is no longer operating at its design condition. Another important effect is the increase in central aperture obstruction (shadow of secondary on primary mirror), particularly if the mirror separation is increased. This effect has a negative effect on the collector function, for it significantly reduces the collection solid angle.

It is now possible to identify a number of questions the answers to which will prove the utility of a modified Schmidt-Cassegrain configuration for LV imaging applications:

1. Can good performance be obtained by simply repositioning the optics without replacing components?

2. What are the required modifications as a function of f-ratio?

3. What is the aberration limit of the modified system as a function of f-ratio?

4. What is the resulting collection solid angle as a function of f-ratio?

5. How well do the modified catadioptric optics perform compared with a similar lens design?

\section{METHODS}

\section{Analytical Tools}

A broad variety of very powerful optical design software is available to assist the optical designer in answering questions such as these (ref. 3). A rigorous prediction of the propagation of the off-axis Gaussian laser beams through the focusing optics (in order to predict the Gaussian-beam-waist diameter and location, astigmatism, and wave-front curvature at the final beam intersection) is possible 
with the more sophisticated physical optics programs. Monochromatic diffraction modulation transfer function (MDMTF) predictions of the imaging capability of the collector are also possible. However, the high cost and time-intensive training required to effectively use these powerful tools makes them less attractive for an isolated problem such as this.

A much easier evaluation of an optical system is possible with geometrical raytracing software. A ray-tracing approach accurately models the refraction and reflection of rays traversing the optical system and may be used to predict the thirdorder Seidel aberrations. Coma, astigmatism, field curvature, and distortion are not significant in this paraxial point-source imaging task of collection. However, attention must be given to spherical aberration, which may be corrected with the aspheric Schmidt corrector element, and chromatic aberration, which may be important for polychromatic operation.

Diffraction effects cannot be predicted with ray-tracing techniques. Gaussianbeam propagation, which is basically a diffraction phenomenon, requires more refined analysis. The simplest approach to predicting Gaussian-beam propagation is to use "thin-lens" equations derived in a manner similar to the thin-lens imaging equations. A somewhat more sophisticated approach, appropriate for both refractive and reflective element analysis, is to model the system with the Gaussianbeam "paraxial" equations. Equations for beam-waist size and location for both the thin-lens and paraxial formulations are presented in the appendix. Neither of these approaches accounts for the astigmatism introduced by off-axis propagation.

However, these predictions are useful as a first approximation of beam-waist size and location.

The analytical approach adopted for this study began with the use of a simple ray-tracing program to analyze modified optical configurations. The particular raytracing software chosen also incorporated a Gauss-Newton optimization algorithm which systematically varied tagged parameters until successive iterations provided an improvement of less than $10^{-6}$ in a target value, typically a point-focus specification. This feature was quite useful in optimizing collector system modifications. The rms blur of ray bundles originating at the axis vertex in the object plane and converging to a best focus in the image plane is used as an indication of the imaging quality of the optical system. Both point focus and confocal backscatter zoom configurations were considered. Finally, an on-axis Gaussian-beam-propagation calcualtion was made using the paraxial equation analysis to evaluate the beam-focusing function.

The real motive for accurately imaging the probe volume is related to improving the signal-to-noise ratio of the instrument. Laser radiation scattered from many sources (e.g., seed material outside the probe volume, beam-focusing optics, test-section windows, and model surfaces that are in the beam path) all contribute noise at the laser wavelength, and this noise cannot be removed by spectral filtering. However, these scattered waves all have substantial transverse 
spatial frequency content and may be reduced by spatially filtering in the image plane (by masking all but the probe-volume image). Aberrations introduced by the imaging system increase the transverse spatial frequency content of the signal radiation, thereby limiting the effectiveness of spatial filtering. With aberrationfree imaging, the spatial filter mask (pinhole) may be matched closely to the sharp probe-volume image to provide highly effective filtering.

\section{Generic f/2 Schmidt Cassegrain Collector}

A quantitative description of a Schmidt-Cassegrain telescope (i.e., mirror apertures, curvatures, and spacing; and corrector-plate surface shape) is necessary if a ray-tracing analysis is to be applied. Unfortunately, the highly competitive market and limited interest in such detailed technical specifications combine to make this proprietary information difficult to obtain. For these reasons a generic SchmidtCassegrain system was examined, with specifications derived in the following manner.

It was observed that most commercial units were geometrically similar regardless of size. It follows that dimensional normalization by some characteristic dimension (e.g., the primary aperture) permits the derivation of a single representative generic design. In keeping with this approach, length specifications appearing herein having no specific units may be assumed to be aperture normalized. One specification that could be obtained for several models and brands of telescope was the focal ratio of the primary mirror, which ranged in value from 2.0 to 2.14. Further observations showed the mirror spacing for the unmodified telescope to be in the range of 1.5, with a Cassegrain focus located approximately 0.75 behind the primary. This information permitted the specification of all but the secondary mirror curvature and the figure of the corrector plate. These were obtained using the optimization feature of the ray tracer as follows. First, the secondary-mirror curvature of an uncorrected infinite conjugate Cassegrain system was optimized to minimize blur. Second, a fourth-order Schmidt corrector plate was optimized for minimum blur at the Fraunhofer C, D, and F $(656.3,589.3$, and $486.1-\mathrm{nm}$ ) wavelengths. This solution (presented in the results section) provided a basic telescope design to be modified for near-focus operation.

\section{Experimental Verification}

Following the prediction phase of this study, a commercial $200-\mathrm{mm}$-aperture Schmidt-Cassegrain telescope was obtained and modified to the predicted optimum configuration for $f / 10$ collection. A simple laboratory exercise was conducted to evaluate the ray-tracer prediction capability and the image quality for the modified telescope collector. A USAF 1951 resolution target was positioned in the object plane. A high-quality (seven-element orthoscopic) astronomical ocular was used to 
image the target. This image was then photographed using eyepiece-projection methods to provide a record of the system imaging capability.

To evaluate the Gaussian-beam focusing function, the telescope was again modified to the predicted optimum configuration. The beam-waist size and location were measured using a translating-slit-type laser-beam profile instrument.

\section{RESULTS AND DISCUSSION}

\section{Infinite Conjugate Schmidt Cassegrain Solution}

Optical specifications for the generic unmodified telescope predicted by the ray tracer are given in table 1. Specifications for the rays used in this solution are given in table 2. Optimization was performed with a target point focus at the image plane. The residual transverse rms blur is a direct indication of total aberration. Certain details, such as the thickness of the corrector plate, have a very limited effect on the focal spot size. A similar optimization with monochromatic rays yielded rms blur of less than $10^{-6}$, indicating an aberration-free design. Therefore, the polychromatic rms blur of $14 \times 10^{-6}$ is an indication of the chromatic aberration limit at infinite conjugate.

A calculation of the diffraction-limited spot size is helpful in determining the relative magnitude of limiting aberrations. The radius of the first minima arising from Fraunhofer diffraction from a circular aperture is given by the expression (ref. 4)

$$
\begin{gathered}
\theta=1.22 \frac{\lambda}{D}=\tan ^{-1}\left(\frac{r}{f}\right) \approx \frac{r}{f} \\
r \approx 1.22 \lambda\left(\frac{f}{D}\right)
\end{gathered}
$$

Although this expression is not strictly applicable to annular aperatures, it provides a useful approximation. For the generic telescope with a limiting aperture located at the corrector plate, the value of $\mathrm{f} / \mathrm{D}$ is 5.25. For an approximate wavelength of $500 \mathrm{~nm}$, the resulting diffraction-limited spot radius is $3.2 \mu$. It follows that the calculated rms chromatic blur is very near the diffraction limit, indicating an excellent optical design. 


\section{Modification for $\mathrm{f} / 10$ Point Focus Collection}

Having acquired a generic model of an aperture-normalized SchmidtCassegrain telescope, it was possible to examine the effect of various modifications and thereby address the questions raised previously. First, what is the best way to modify the telescope to operate at close range? Of the possible modifications, a simple repositioning of the three optical elements is certainly the easiest to accomplish. Normal focusing at close range is accomplished by translating the primary mirror back as far as possible (increasing mirror spacing). It follows that a repositioning of the primary mirror by extending the telescope tube will bring the near focus in even closer. An additional degree of freedom in component positioning can be obtained if the mounting of the secondary mirror is modified to permit independent positioning of the secondary mirror with respect to the corrector plate.

Replacing one or more of the components is another possible way to obtain a closer focus. Replacing the large-aperture corrector plate or primary mirror would not be cost effective. But it is possible that the secondary mirror could be replaced with a stock convex spherical mirror or a with silvered plano-convex or bi-convex lens.

These possible modifications were examined using ray-tracing methods. Both tube-extension and repositioning of the secondary were optimized for monochromatic 514-nm (argon laser output) operation for a probe-volume-to corrector-plate spacing of 10 times the primary aperture ( $f / 10)$. Rays from a pointsource object were targetted to a point focus in the image plane. The resulting optical configurations are presented in table 3 . The minimized rms blur values are an indication of residual aberration. Significant spherical aberration is introduced with the simple tube-extension approach. But if the secondary mirror is also repositioned, aberrations may be virtually eliminated.

The optimum positioning of components places the primary mirror a distance of about twice the primary aperture behind the corrector. This increased separation reduces the collection solid angle and increases the size of the collector package. Therefore, a third optimization with tube-extension and a fixed secondary position, but variable secondary curvature, is also explored. Table 3 indicates that this combination also provides near zero aberration with a somewhat shorter tube. Of course it is not possible to exactly match this secondary-mirror curvature with a stock mirror or even with a reflectively coated lens, but a value near this will decrease the tube length somewhat, as shown in line 4 of table 3 . In practice one would need to select a replacement secondary with curvature as close as possible to the optimum and then re-optimize the primary and replacement secondary mirror positions.

These results indicate two practical approaches to telescope modification: (1) repositioning both the primary and secondary mirrors by extending the telescope 
tube and modifying the secondary mount, and (2) repositioning the primary mirror by increasing the tube length and replacing the secondary mirror.

\section{Polychromatic Operation}

Because rays encounter the nearly flat corrector plate surface at low angles of incidence, dispersion effects and, therefore, chromatic aberrations are minimal. Since two-color operation is common with LV systems, chromatic aberration is important. Accordingly, the system was optimized for several f-ratios for rays at wavelengths of both 514 and $488 \mathrm{~nm}$ (the strongest argon-ion laser lines). The rms blur was again essentially zero, as seen in lines 5 and 6 of table 3 , indicating very low chromatic aberration at these closely spaced wavelengths. These results indicate that a properly modified telescope collector may be expected to give polychromatic performance (at argon wavelengths) near the diffraction limit for f-ratios near 10.

\section{Performance As a Function of f-Ratio}

In practice, the f-ratio will vary for each unique optical configuration. Having identified two possible approaches to telescope modification, a systematic ray-tracing evaluation of the performance of modified configurations as a function of $f$-ratio was made. Optical element positions and curvatures were optimized, keeping the Cassegrain focus a constant 0.75 aperture units behind the primary mirror. Optimization was done dichromatically at wavelengths of 514 and $488 \mathrm{~nm}$ with rays of equal angular separation. The results of this evaluation are presented in table 4 and plotted in figures $4-6$. Near-zero aberration was achieved for all cases.

Figure 4 shows the required element positioning as a function of f-ratio for the primary-and secondary-repositioning modification procedure. It may be seen that the required increase in tube length does not exceed the aperture for f-ratios above 6 . Figure 5 shows the required element positioning as a function of $f$-ratio for the second (primary repositioning and secondary replacement) modification procedure. A slightly shorter assembly results.

The collection solid angle is an important factor in determining the collected light intensity at the photodetector. The effects of $\mathrm{f}$-ratio on collection solid angle for the two modification schemes are shown in figure 6 . The collection solid angle for a lens collector is also plotted for comparison. The lens collector clearly provides the best possible collection solid angle for a given aperture. The required extension of the telescope tube removes the limiting aperture of the primary mirror an additional distance from the probe volume, thereby reducing the collection solid angle. The shadow cast by the secondary on the primary mirror further reduces the effective solid angle. These losses increase significantly with decreasing f-ratio. The curves for the two modifications are reasonably close over the entire range. 
It is anticipated that these results may be directly applied by the reader in specifying a telescope modification for his specific collector application. Once an $\mathrm{f}$ ratio has been selected (based on telescope aperture and operating range), the aperture-normalized component spacings in table 3 may be used to approximate the required tube extension and secondary-mirror remounting apparatus (or replacement secondary-mirror curvature).

\section{Comparison with a Lens Collector}

Now it is possible to make some comparisons of modified telescopic collector and a lens collector. A lens provides a larger collection solid angle for a given aperture size, but does a lens focus the collected light as well? To help answer this question, two commercially available lenses were evaluated. The accessibility of technical data on many commercial lenses permited them to be easily analyzed with the ray tracer. The first lens, designated L1, is an air-spaced, doublet laser aplanat corrected for spherical aberration and coma but not for axial color. A large stock lens $(100-\mathrm{mm}$ aperture, $500-\mathrm{mm}$ focal length, $\mathrm{f} / 5)$ was selected. The second lens, L2, is a computer-optimized, cemented achromatic doublet designed according to the optimum MTF criteria for quality imaging applications. Again, a large lens (150-mm aperture, $1000-\mathrm{mm}$ focal length, f/6.67) was selected. Each of these lenses is designed for infinite-conjugate-ratio operation. For unity conjugate ratio, two lenses are placed back to back.

The results of the ray-tracer analysis are presented in table 5 for both monochromatic (514-nm) and dichromatic (514-, 488-nm) operation. Results are reported in (lens) aperture-normalized units. Lens L1 provides the lowest rms blur for monochromatic operation but shows significant axial color aberration. Lens L2 has more spherical aberration at the single wavelength, but lower chromatic aberration. These results are in keeping with the design criteria for the two lenses. These stock lenses do not perform near the diffraction limit and will not match the modified telescope performance.

It is generally possible to optimize a custom lens to perform near the diffraction limit at two specific wavelengths, particularly if the f-ratio is large. Such a design requires the expertise and design tools of an experienced optical engineer. Custom manufacturing and testing procedures are also required. These factors all contribute to the high cost of custom lens systems.

An additional attractive feature of the modified telescope collector is that the primary mirror can be focussed, thereby changing the f-ratio. This would be useful in an LV system that was required to operate at several different ranges. A singlelens collector cannot be focused. A zoom lens system could be designed with such a capability but this would add another dimension of complexity to the design and would further degrade single-point performance, since performance would be optimized over a range. 


\section{Confocal Backscatter}

In the confocal backscatter configuration shown in figure 3, laser-beam focusing and backscattered-light collection are performed by the same optical system. The laser output is divided into two parallel beams, which are aligned parallel to the optical axis of the Cassegrain system. The Cassegrain optics focus these beams to an intersection that defines the probe volume. Backscattered light is collected and collimated by the Cassegrain optics. A mirror with holes in it (to pass the outgoing laser beams) may be used to direct the back-scattered light to a lens and focus it onto a photodetector.

This configuration is useful when optical access is available from one side only. Alignment stability and alignability are quite good. However, backscatter operation suffers from low back-scattering intensity and from noise contamination from the outgoing laser beams. This noise may arise from scattering from optical surfaces, or from model surface glare (in the case of boundary-layer measurements). These noise effects may be minimized if well-corrected optics are used. If the beamfocusing task is accomplished so as to provide very high energy densities at the probe volume, the signal power will increase and the spatial frequency distribution of the signal photons will narrow. Well-corrected collection permits very narrow spatial filtering of the collected light just ahead of the photodetector (by means of a pinhole) to reject unwanted optical noise from optical-surface scatter or glare.

The required modifications to the Schmidt-Cassegrain telescope were again computed by using the ray-tracing algorithm and a collector mode of operation. Rays emanating from a point source were traced through the system. Optical element positions were optimized to provide collimated light (exiting from the optimization routine when subsequent iterations changed the direction cosine of the collimated light by less than 1 in $10^{-6}$.) These solutions are presented in table 6 for the same two modification schemes that were considered for point-focus collection: (1) primary and secondary mirror re-positioning, and (2) primary mirror re-positioning and secondary-mirror replacement. The performances of the modified optical systems are quite similar to that of the point-focus case. Near-zero aberration is achieved for either modification scheme.

\section{Laser Beam Focusing}

The capability of the above confocal backscatter solutions to focus the outgoing laser beams to a high-energy-density probe volume was evaluated using the paraxial formulations given in the appendix. An effective corrector plate focal length computed from the curvature of the fourth-order surface shape at the radius of the beam centroid was used. The paraxial formulas for Gaussian-beam propagation (see appendix) were used to predict and compare the performance of the two lenses 
discussed in the preceding section with the performance of modified generic confocal backscatter telescope configurations having the same aperture and f-ratio. Since Gaussian-beam propagation is scale dependent, the aperture-normalized solutions were converted to physical length units by multiplying by the aperture of the comparison lens (100 $\mathrm{mm}$ for L1, $150 \mathrm{~mm}$ for L2). Calculations were made for a laser wavelength of $514.5 \mathrm{~nm}$.

These data (table 7) indicate that the modified telescope optics provide a smaller beam-waist than either of the lenses. This improvement is principally owing to the initial expansion of the beam by the negative secondary-mirror element. This provides a large beam diameter at the primary mirror, which then provides a large convergence angle (and therefore a small waist diameter) at the final probe-volume waist. Similar performance could be obtained for a lens system consisting of a negative (plano-concave) lens followed by back-to-back infinite conjugate aplanats, as shown in the final line of table 7.

The Rayleigh range, which corresponds to the region of maximum wave-front curvature, is also tabulated. In order to ensure that wave fronts are planar in the probe volume (to avoid probe-volume fringe curvature), it is necessary to place the beam waist at the beam intersection to within a small fraction $(\sim 10 \%)$ of the Rayleigh range. The predicted axial beam-waist location does not coincide with the crossing for any but the L1 laser aplanat. This problem is often encountered, even with custom-designed lenses. A common solution involves the insertion of a beam collimating telescope in front of the beamsplitter to permit fine tuning of the beam waist location.

\section{Experimental Verification}

A 200-mm-aperture telescope was obtained and modified to evaluate the modification methods proposed. The unit was disassembled and component specifications measured by various means, including a spherometer, parallel laser beam imaging, and interferometry (for the corrector plate figure). These specifications were then used to predict the required element spacings and best performance for operation at a range of $2 \mathrm{~m}$. Both point-focus and confocal backscatter solutions were obtained. The confocal backscatter solution required the replacement of the secondary mirror with a silvered lens. These solutions were configured on the optics bench and evaluated as described previously.

Alignment can be critical for multi-element optical systems operating near the diffraction limit. Astronomical telescopes are factory-aligned in their infinite conjugate mode. But the corrector plate and secondary mirror had to be removed and repositioned. It was therefore necessary to devise a procedure for re-aligning the modified system. For first-surface spherical optics, centration may be achieved through tilt adjustment; therefore, there were only four alignment adjustments to consider: corrector tilt, corrector centration, primary tilt, and secondary tilt. 
The corrector plate is a very weak element and not likely to require rigorous alignment. This assumption was examined using ray tracing and it was found that for a centration error of $1 \mathrm{~mm}$, the dichromatic rms blur rose to only $10 \mu$. A tilt misalignment of $1^{\circ}$ produced negligible aberration. It may be concluded that for an accurately machined tube extension, the alignment of the corrector is not critical. The primary-mirror mounting to the focusing mechanism need not be disturbed during the modification process and may be assumed to remain within the factoryprescribed limits. Finally, the secondary mirror mount was already fitted with a tilt adjustment that was preserved in the modified secondary mount design to permit final alignment.

The final alignment procedure paralleled the recommended procedure for telescope alignment. A tiny point source consisting of a 10- $\mu$ pinhole in front of a light bulb was imaged through the telescope with a high quality astronomical ocular and centered in the field of view. The image was defocused slightly so that the central obstruction shadow was visible. The secondary mirror tilt was then adjusted so that the central obstruction shadow, the image periphery, and the field of view were all concentric. This required several iterations between secondary tilt adjustment and pointing of the entire telescope assembly.

The imaging capability of the point-focus-collector configuration was evaluated by placing a USAF 1951 resolution target in the image plane. A high-quality astronomical ocular was used for high-magnification imaging of this target ruling. Figure 7 represents a photograph of this image taken with a $35-\mathrm{mm}$ camera using the common eyepiece projection method of astrophotography. The group-seven, element-two ruling (144 lines/mm, $7-\mu$ spacing) was marginally resolved, indicating that the entire optical system (including the ocular) is imaging very near the diffraction limit.

As final proof of the usefulness of this approach, several of these modified telescope point-focus collectors have been installed and are functioning quite well in a 2-m-range fixed-focus LV system.

To evaluate the laser-beam focusing function, the telescope was configured to the f-10 collimated-collection configuration. A helium-neon laser output was divided into two equal-intensity, parallel beams. The telescope was aligned as shown in figure 3 , with beam spacing adjusted to locate the beam centroid at $80 \%$ of the primary-mirror (limiting aperture) radius. The telescope was positioned $2.2 \mathrm{~m}$ from the laser output waist. The beam profile and diameter $\left(d f^{\prime \prime \prime}\right)$ were measured in the lens focal plane (at a distance $z f^{\prime \prime \prime}$ from the corrector plate) by means of a spinning-slit optical profiler. Additionally, the beam-waist location $\left(z_{0}{ }^{\prime \prime \prime}\right)$ was measured by translating the profiler along the z-axis to locate the minimum diameter $\left(d_{0}^{\prime \prime \prime}\right)$. 
These data are presented in table 8 . The predicted and measured values for beam-waist diameter and location agree quite well, considering the simplicity of the prediction model. A 1.667X collimating telescope was inserted between the laser and beamsplitter to adjust the beam waist to coincide with the intersection and the waist diameter was again measured. Beam-waist data are in poorer agreement here, possibly owing to some error in the assembly of the beam-collimating telescope (such as a reversed lens).

As previously stated, the approach to predicting the beam-waist size and location for the reflective elements of the modified telescope utilized the paraxial Gaussian-beam equations, neglecting the astigmatism introduced by off-axis propagation. Beam-waist measurements showed negligible astigmatism, indicating that this was a reasonable assumption. The corrector plate was modeled as a simple plano lens with an effective focal length determined by the front-surface curvature at the beam centroid radius. In the thin-lens prediction model, this final weak element had a minor effect on the final waist, depending on the radial location of the beam centroid.

This condition brought up the possibility of removing the corrector plate altogether for the beam-focusing operation. This option was explored, but was found to result in extreme astigmatism at the waist. These results indicate that the corrector plate performs the important function of removing much of the astigmatisn introduced by off-axis incidence on the spherical mirrors.

\section{CONCLUSIONS}

This work has explored the possibility of adapting commercially available Schmidt-Cassegrain telescope optics to the laser beam focusing and scattered-light collection tasks of medium-to-long-range LV work. The specific objective was to determine if these very well corrected yet relatively inexpensive infinite conjugate optical systems could be easily adapted for operation at near-unity conjugate ratio. A generic, aperture-normalized Schmidt-Cassegrain system was specified based on an average of the specifications for several commercial units. Modifications to this generic system were analytically examined using geometric ray tracing, and the paraxial Gaussian-beam propagation formulations.

These prediction methods indicated that very good performance could be obtained for f-ratios as low as 4.0. Two relatively simple modification procedures were identified that maintained diffraction limited performance.

1. The three active elements (primary mirror, secondary mirror, and corrector plate) may be independently repositioned. This requires a lengthening of the telescope tube and modification of the secondary mirror mount. 
2. The primary mirror may be re-located and the secondary mirror may be replaced. This again requirs a lengthening of the tube and replacement of the secondary mirror with a stock convex mirror or a reflectively coated lens of the correct curvature.

The predicted performance of these modified telescope systems was compared with the predicted performance of large-aperture commercial lenses. The probevolume imaging capability of the modified telescopes was superior to that of the lenses for both monochromatic and dichromatic operation. Because of the central obstruction of the secondary mirror and the separation of the primary mirror from the corrector plate, the collection solid angle for the modified telescope systems was considerably less than that of an equivalent aperture lens, particularly at low f-ratio. However, the excellent imaging capability and large apertures of the modified telescopes offset this limitation.

The modified telescope also performed the laser beam focusing task quite well. The negative secondary-mirror/positive-primary mirror combination provided a very small (high energy density) beam waist near the probe volume. The beamwaist and beam-intersection points do not coincide (as is generally the case in dualbeam LV). However, this problem may be treated with additional beam telescope optics upbeam of the beamsplitter to fine tune the beam-waist location.

These promising predictions were validated with hardware in the laboratory. A $200-\mathrm{mm}$ aperture commercial unit was purchased and modified to the predicted optimum configurations for both point collection and dual-beam focusing. Imaging resolution and beam-waist diameter and location measurements agreed well with the predictions and prove the utility of this approach in the optical imaging and beam-manipulation tasks of LV work. Several modified telescope collectors are functioning in a long-range LV system. 


\section{APPENDIX}

\section{THIN LENS AND PARAXIAL GAUSSIAN BEAM EQUATIONS}

The following relationships are from reference 2 .

Relationship between beam-waist diameter and beam divergence for a Gaussian TEM $_{00}$ mode beam:

$$
d_{0}=\frac{4 \lambda}{\pi \theta}
$$

Definition of the Raleigh range and its relation to beam-waist diameter and beam divergence:

$$
\mathrm{z}_{\mathrm{R}}=\frac{\mathrm{d}_{\mathrm{o}}}{\theta}=\frac{4 \lambda}{\pi \theta^{2}}=\frac{\pi \mathrm{d}_{0}^{2}}{4 \lambda}
$$

Thin-lens formulas for the prediction of beam-waist diameter and location:

$$
\begin{gathered}
\alpha=\frac{|f|}{\left[\left(z_{2}-f\right)^{2}+z_{R}^{2}\right]^{\frac{1}{2}}} . \\
d_{0}^{\prime}=\left[\frac{f^{2} d_{0}^{2}}{\left(z_{2}-f\right)^{2}+z_{R}^{2}}\right]^{\frac{1}{2}}=\alpha d_{0} \\
z_{R}{ }^{\prime}=\alpha^{2} z_{R} \\
z_{1}^{\prime}=f+\alpha^{2}\left(z_{2}-f\right)=f+\left[\frac{\theta}{\alpha}\right. \\
\left.\frac{f^{2}\left(z_{2}-f\right)}{\left(z_{2}-f\right)^{2}+z_{R}^{2}}\right]
\end{gathered}
$$

The beam diameter at any $z$-location may be calculated from: 


$$
d=d_{0}\left[1+\left(\frac{\theta z}{d_{0}}\right)^{2}\right]^{\frac{1}{2}}
$$

Paraxial formulas for the calcualtion of beam propagation through interfaces having optical power:

$$
\begin{gathered}
\phi=\left(n^{\prime}-n\right) / r \\
\alpha=\frac{\left(n n^{\prime}\right)^{\frac{1}{2}}}{\left[\left(n-z_{2} \phi\right)^{2}+z_{R}{ }^{2} \phi^{2}\right]^{\frac{1}{2}}}
\end{gathered}
$$

Beam-waist diameter, Raleigh range, divergence, and waist location are again given by

$$
\begin{gathered}
\mathrm{d}_{0}^{\prime}=\alpha \mathrm{d}_{0} \\
\mathrm{z}_{\mathrm{R}}{ }^{\prime}=\alpha^{2} \mathrm{z}_{\mathrm{R}} \\
\theta^{\prime}=\frac{\theta}{\alpha} \\
\mathrm{z}_{1}=\mathrm{f}+\alpha^{2}\left(\mathrm{z}_{2}-\mathrm{f}\right)
\end{gathered}
$$




\section{REFERENCES}

1. Durst, F.; Melling, A.; and Whitelaw, J. H.: Principles and Practice of LaserDoppler Anemometry, Second ed. Academic Press, New York, 1981, p. 102.

2. O'Shea, D. C.: Elements of Modern Optical Design. John Wiley \& Sons, New York, 1985, p. 217.

3. Kidger, M. J.: Personal Computers Facilitate Optical Design. Laser Focus, May 1987, pp. 86-94.

4. Jenkins, F. A.; and White, H. E.: Fundamentals of Optics. McGraw-Hill, New York, 1965, p. 329. 
TABLE 1. - GENERIC SCIMMIDT-CASSEGRAIN TELESCOPE SPECIFICATION, INFINITE CONJUGATE RATIO, APERTURE NORMALIZED UNITS

\begin{tabular}{|l|l|l|}
\hline Corrector plate & Material & BK7 \\
Figuring depth & Thickness & 0.01500 \\
& d=0.001803 $\mathrm{r}^{2}-0.005382 \mathrm{r}^{4}$ & \\
& Location (front) & 0.000000 \\
\hline Primary mirror & Curvature & 0.25000 \\
& Location & 1.50000 \\
\hline Secondary mirror & Curvature & 0.76138 \\
& Location & 0.01500 \\
\hline Cassegrain Focus & Location $\quad$ C, D, and F lines & 0.25000 \\
& RMS Blur; & 0.000014 \\
\hline
\end{tabular}

TABLE 2. - GENERIC SCHMIDT-CASSEGRAIN TELESCOPE RAY TABLE, INFINITE CONJUGATE RATIO, APERTURE NORMALIZED UNITS.

\begin{tabular}{|l|r|r|r|}
\hline$r_{0}$ & \multicolumn{3}{|c|}{$r_{\text {ocus }}\left(\times 10^{-6}\right)$} \\
\hline & $\lambda=656.3(\mathrm{C})$ & $\lambda=589.3(\mathrm{D})$ & $\lambda=486.1(\mathrm{~F})$ \\
\hline 0.21517 & 12.660 & 0.761 & -27.189 \\
0.28868 & 15.121 & 4.020 & -22.057 \\
0.34695 & 13.234 & 5.754 & -11.814 \\
0.39675 & 6.410 & 4.582 & 0.289 \\
0.44096 & -5.977 & -0.511 & 12.330 \\
0.48113 & -24.523 & -10.350 & 22.942 \\
\hline
\end{tabular}

TABLE 3. - MODIFIED TELESCOPE CONFIGURATIONS FOR F/10 OPERATION; APERTURE NORMALIZED UNITS a

\begin{tabular}{|c|c|r|r|r|r|}
\hline Config. & $\begin{array}{c}\text { Primary } \\
\text { location }\end{array}$ & $\begin{array}{c}\text { Secondary } \\
\text { location }\end{array}$ & $\begin{array}{c}\text { Secondary } \\
\text { curvature }\end{array}$ & $\begin{array}{c}\text { Cassegrain } \\
\text { focus }\end{array}$ & $\begin{array}{l}\text { Rms } \\
\text { blur }\end{array}$ \\
\hline 1 & 1.500 & 0.015 & -0.76138 & 2.250 & 0.000014 \\
2 & 1.888 & 0.015 & -0.76138 & 2.638 & 0.000286 \\
3 & 2.358 & 0.503 & -0.76138 & 3.018 & 0.000000 \\
4 & 2.028 & 0.015 & -1.14144 & 2.778 & 0.000001 \\
5 & 2.356 & 0.501 & -0.76138 & 3.016 & 0.000002 \\
6 & 2.028 & 0.015 & -1.13911 & 2.778 & 0.000003 \\
\hline
\end{tabular}

a Configurations: (1) Basic telescope solution; (2) Reposition primary mirror;

(3) Reposition primary and secondary mirrors; (4) Reposition primary mirror, replace secondary mirror; (5) Dichromatic performance for config. 3; (6)

Dichromatic performance for config. 4; 
TABLE 4. - MODIFIED SCHMIDT-CASSEGRAIN POINT COLLECTOR; OPTIMUM CONFIGURATION AS A FUNCTION OF F-RATIO; APERTURE NORMALIZED UNITS a

\begin{tabular}{|c|c|c|c|c|c|}
\hline & & & & \\
\hline $\begin{array}{c}\text { Collection } \\
\text { F-Ratio }\end{array}$ & $\begin{array}{c}\text { Primary } \\
\text { Location }\end{array}$ & $\begin{array}{c}\text { Secondary } \\
\text { Location }\end{array}$ & $\begin{array}{c}\text { Secondary } \\
\text { Curvature }\end{array}$ & $\begin{array}{c}\text { Cassegrain } \\
\text { Focus }\end{array}$ & $\begin{array}{c}\text { RMS } \\
\text { Blur }\end{array}$ \\
\hline \multicolumn{6}{|c|}{} \\
\hline \multicolumn{6}{|c|}{ Modification 1. Primary and secondary mirror repositioning } \\
\hline 4.0 & 2.73628 & 0.43659 & 0.76138 & 3.48628 & 0.000003 \\
5.0 & 2.61071 & 0.43934 & 0.76138 & 3.36071 & 0.000003 \\
6.0 & 2.52595 & 0.45175 & 0.76138 & 3.27595 & 0.000003 \\
7.0 & 2.46512 & 0.46567 & 0.76138 & 3.21512 & 0.000002 \\
8.0 & 2.41952 & 0.47884 & 0.76138 & 3.16952 & 0.000002 \\
9.0 & 2.38419 & 0.49068 & 0.76138 & 3.13419 & 0.000002 \\
10.0 & 2.35613 & 0.50122 & 0.76138 & 3.10613 & 0.000002 \\
11.0 & 2.33340 & 0.51058 & 0.76138 & 3.08340 & 0.000002 \\
12.0 & 2.31470 & 0.51895 & 0.76138 & 3.06470 & 0.000002 \\
13.0 & 2.29910 & 0.52647 & 0.76138 & 3.04910 & 0.000002 \\
15.0 & 2.27471 & 0.53946 & 0.76138 & 3.02471 & 0.000002 \\
20.0 & 2.23833 & 0.56468 & 0.76138 & 2.98833 & 0.000003 \\
\hline \multicolumn{7}{|c}{} \\
Modification 2. Primary mirror repositioning, secondary mirror replacement \\
\hline 4.0 & 2.52179 & 0.01500 & 1.18737 & 3.27179 & 0.000005 \\
5.0 & 2.38146 & 0.01500 & 1.18727 & 3.13146 & 0.000005 \\
6.0 & 2.27620 & 0.01500 & 1.18630 & 3.02620 & 0.000005 \\
7.0 & 2.19402 & 0.01500 & 1.17979 & 2.94402 & 0.000004 \\
8.0 & 2.12790 & 0.01500 & 1.16866 & 2.87790 & 0.000004 \\
9.0 & 2.07346 & 0.01500 & 1.15460 & 2.82346 & 0.000003 \\
10.0 & 2.02782 & 0.01500 & 1.13911 & 2.77782 & 0.000003 \\
11.0 & 1.98898 & 0.01500 & 1.12322 & 2.73898 & 0.000003 \\
12.0 & 1.95552 & 0.01500 & 1.10750 & 2.70552 & 0.000003 \\
13.0 & 1.92639 & 0.01500 & 1.09238 & 2.67639 & 0.000003 \\
15.0 & 1.87815 & 0.01500 & 1.06452 & 2.63815 & 0.000003 \\
20.0 & 1.79522 & 0.01500 & 1.00949 & 2.54522 & 0.000003 \\
\hline \multicolumn{7}{|c|}{}
\end{tabular}

acorrector plate location and figure, primary curvature, and primary-to-Cassegrain focus spacing all constant (see Table 1) 
TABLE 5 - RESULTS OF RAY TRACER ANALYSIS FOR LENSES L1 AND L2 a

\begin{tabular}{|c|c|c|c|c|}
\hline & Surface & Glass & $\begin{array}{c}\text { Radius } \\
\text { (meters) }\end{array}$ & $\begin{array}{c}\text { Thickness } \\
\text { (meters) }\end{array}$ \\
\hline \multicolumn{5}{|c|}{ L1 } \\
\hline & point source & air & & 5.00000 \\
\hline 2 & first lens & SF11 & 25.00000 & 0.08000 \\
\hline 3 & first lens & air & 5.89677 & 0.05304 \\
\hline 4 & first lens & SF11 & 8.86101 & 0.12000 \\
\hline 5 & first lens & air & -3.68171 & 0.00000 \\
\hline 6 & second lens & SF11 & 3.68171 & 0.12000 \\
\hline 7 & second lens & air & -8.86101 & 0.05304 \\
\hline 8 & second lens & SF11 & -5.89667 & 0.08000 \\
\hline & second lens & air & -25.00000 & $\sim 5.0$ \\
\hline \multirow{3}{*}{\multicolumn{2}{|c|}{$\begin{array}{l}\text { best focus } \\
\text { monochromatic }(514 \mathrm{~nm}) \\
\text { dichromatic }(514,488 \mathrm{~nm})\end{array}$}} & & RMS blur & distance \\
\hline & & & 0.000013 & 4.93800 \\
\hline & & & 0.002741 & 4.91622 \\
\hline \multicolumn{5}{|c|}{ L2 } \\
\hline & point source & \multirow{4}{*}{$\begin{array}{l}\text { SF5 } \\
\text { SK11 }\end{array}$} & & 6.66667 \\
\hline & first lens & & 12.74660 & 0.06667 \\
\hline 3 & first lens & & 3.06940 & 0.09753 \\
\hline 4 & first lens & & -4.23887 & 0.00000 \\
\hline 5 & second lens & \multirow{2}{*}{$\begin{array}{l}\text { SK11 } \\
\text { SF5 }\end{array}$} & 4.23887 & 0.09753 \\
\hline & second lens & & -3.06940 & 0.06667 \\
\hline \multirow{2}{*}{\multicolumn{2}{|c|}{$\begin{array}{l}7 . \quad \text { second lens } \\
\text { hect focuc }\end{array}$}} & SF5 & -12.74660 & $\sim 6.67$ \\
\hline \multirow{3}{*}{\multicolumn{2}{|c|}{$\begin{array}{l}\text { monochromatic }(514 \mathrm{~nm}) \\
\text { dichromatic }(514,488 \mathrm{~nm}) \\
\end{array}$}} & & RMS blur & distance \\
\hline & & & 0.000069 & 6.74579 \\
\hline & & & 0.000119 & 6.74693 \\
\hline
\end{tabular}

a L1: Airspaced laser aplanat lens collector set, best focus configuration, aperture normalized units, aperture $=100 \mathrm{~mm}$; L2: MTF computer optimized achromatic cemented doublet lens collector set, best focus configuration, aperture normalized units, aperture $=150 \mathrm{~mm}$. 
TABLE 6. - MODIFIED SCHMIDT-CASSEGRAIN LASER BEAM FOCUSING; OPTIMUM CONFIGURATION AS A FUNCTION OF F-RATIO; APERTURE NORMALIZED UNITS

\begin{tabular}{|c|c|c|c|c|c|}
\hline F-Ratio & $\begin{array}{l}\text { Primary } \\
\text { Location }\end{array}$ & $\begin{array}{c}\text { Secondary } \\
\text { Location }\end{array}$ & $\begin{array}{l}\text { Secondary } \\
\text { Curvature }\end{array}$ & $\begin{array}{c}\text { Cassegrain } \\
\text { Focus }\end{array}$ & $\begin{array}{l}\text { RMS } \\
\text { Blur }\end{array}$ \\
\hline \multicolumn{6}{|c|}{ Modification 1. Primary and secondary mirror repositioning } \\
\hline 4.0 & 2.78160 & 0.60617 & 0.76138 & 3.53160 & 0.000001 \\
\hline 5.0 & 2.66250 & 0.61806 & 0.76138 & 3.41250 & 0.000001 \\
\hline 6.0 & 2.58565 & 0.64078 & 0.76138 & 3.33565 & 0.000001 \\
\hline 7.0 & 2.53357 & 0.66547 & 0.76138 & 3.28357 & 0.000001 \\
\hline 8.0 & 2.49727 & 0.68963 & 0.76138 & 3.24727 & 0.000001 \\
\hline 9.0 & 2.47161 & 0.71257 & 0.76138 & 3.22161 & 0.000001 \\
\hline 10.0 & 2.45348 & 0.73424 & 0.76138 & 3.02348 & 0.000001 \\
\hline 11.0 & 2.44086 & 0.75474 & 0.76138 & 3.19086 & 0.000001 \\
\hline 12.0 & 2.43244 & 0.77428 & 0.76138 & 3.18244 & 0.000001 \\
\hline 13.0 & 2.42722 & 0.79296 & 0.76138 & 3.17722 & 0.000001 \\
\hline 15.0 & 2.42381 & 0.82820 & 0.76138 & 3.17381 & 0.000001 \\
\hline 20.0 & 2.44078 & 0.90893 & 0.76138 & 3.19078 & 0.000001 \\
\hline \multicolumn{6}{|c|}{ Modification 2. Primary mirror repositioning, secondary mirror replacemen } \\
\hline 4.0 & 2.50432 & 0.01500 & 1.26981 & 3.25432 & 0.000002 \\
\hline 5.0 & 2.36210 & 0.01500 & 1.27223 & 3.11210 & 0.000002 \\
\hline 6.0 & 2.25499 & 0.01500 & 1.27204 & 3.00499 & 0.000002 \\
\hline 7.0 & 2.17090 & 0.01500 & 1.26547 & 2.92090 & 0.000002 \\
\hline 8.0 & 2.10285 & 0.01500 & 1.25387 & 2.85285 & 0.000002 \\
\hline 9.0 & 2.04646 & 0.01500 & 1.23918 & 2.79646 & 0.000002 \\
\hline 10.0 & 1.99889 & 0.01500 & 1.22303 & 2.74889 & 0.000002 \\
\hline 11.0 & 1.95817 & 0.01500 & 1.20647 & 2.70817 & 0.000002 \\
\hline 12.0 & 1.92289 & 0.10500 & 1.19013 & 2.67289 & 0.000002 \\
\hline 13.0 & 1.89200 & 0.01500 & 1.17443 & 2.64200 & 0.000002 \\
\hline 15.0 & 1.84048 & 0.01500 & 1.14556 & 2.59048 & 0.000002 \\
\hline 20.0 & 1.75065 & 0.01500 & 1.08879 & 2.50065 & 0.000002 \\
\hline
\end{tabular}


TABLE 7. - PROBE VOLUME BEAM WAIST DIAMETER AND LOCATION PREDICTIONS a

\begin{tabular}{|c|c|c|c|c|c|}
\hline Optical system & \multicolumn{5}{|c|}{ Probe volume beam properties } \\
\hline & $\begin{array}{l}d_{0}{ }^{\prime \prime \prime} \\
(\mathrm{mm})\end{array}$ & $\begin{array}{l}z_{0}{ }^{\prime \prime \prime} \\
(\mathrm{mm})\end{array}$ & $\begin{array}{l}z_{f}^{\prime \prime \prime} \\
(\mathrm{mm})\end{array}$ & $\begin{array}{l}z_{R}^{\prime \prime \prime \prime} \\
(\mathrm{mm})\end{array}$ & $\begin{array}{l}\left(z_{0}{ }^{\prime \prime}{ }^{\prime}-z_{f}{ }^{\prime \prime \prime}\right) / z_{R}{ }^{\prime \prime \prime} \\
\text { (nondimensional) }\end{array}$ \\
\hline f/5 Telescope modification & 0.042 & 508 & 500 & 3 & 2.667 \\
\hline Lens L1 laser aplanat & 0.209 & 502 & 500 & 66 & 0.030 \\
\hline f/6.67 Telescope modification & 0.082 & 1029 & 1000 & 10 & 2.900 \\
\hline Lens L2 computer opt. achromat & 0.467 & 1115 & 1000 & 333 & 0.345 \\
\hline f / 5 Negative-positive lens system & 0.054 & 502 & 500 & 5 & 0.400 \\
\hline
\end{tabular}

a Initial beam properties: Wavelength $\lambda=514.5 \mathrm{~nm}$; waist diameter $d_{0}=1.3 \mathrm{~mm}$; divergence $\theta=0.5 \mathrm{mrad}$; Rayleigh range $z_{R}=2.617 \mathrm{M}$; waist location $z_{2}=2.0 \mathrm{M}$ upbeam of first optical surface.

TABLE 8. - PROBE VOLUME BEAM WAIST DIAMETER AND LOCATION MEASUREMENTS a

\begin{tabular}{|l|l|l|l|l|l|l|}
\hline & \multicolumn{6}{|c|}{ Laser beam crossing and waist dimensions } \\
\hline & $\begin{array}{l}d_{f}^{\prime \prime \prime} \\
(\mathrm{mm})\end{array}$ & $\begin{array}{c}z_{f}^{\prime \prime \prime} \\
(\mathrm{mm})\end{array}$ & $\begin{array}{c}d_{0}{ }^{\prime \prime} \\
(\mathrm{mm})\end{array}$ & $\begin{array}{c}z_{0}{ }^{\prime \prime} \\
(\mathrm{mm})\end{array}$ & $\begin{array}{c}z_{R}{ }^{\prime \prime \prime} \\
(\mathrm{mm})\end{array}$ & $\begin{array}{c}\left.z_{0}^{\prime \prime \prime}-z_{f}^{\prime \prime \prime}\right) / z_{R}{ }^{\prime \prime \prime} \\
(\text { nondimensional) }\end{array}$ \\
\hline predicted & 0.335 & 2000 & 0.180 & 2086 & 11.2 & 7.678 \\
measured & 0.316 & 2000 & 0.187 & 2066 & & \\
predicted, with beam telescope & 0.142 & 2000 & 0.142 & 2000 & 25.0 & 0.000 \\
measured, with beam telescope & 0.190 & 2000 & 0.190 & 2000 & & \\
\hline
\end{tabular}

a Initial beam properties: Wavelength $\lambda=632.8 \mathrm{~nm}$; waist diameter $d_{0}=1.1 \mathrm{~mm}$; divergence $\theta=0.73 \mathrm{mrad}$; Rayleigh range $z_{R}=1.50 \mathrm{M}$; laser waist location $z_{2}=2.16$ $M$ upbeam of first optical surface. 


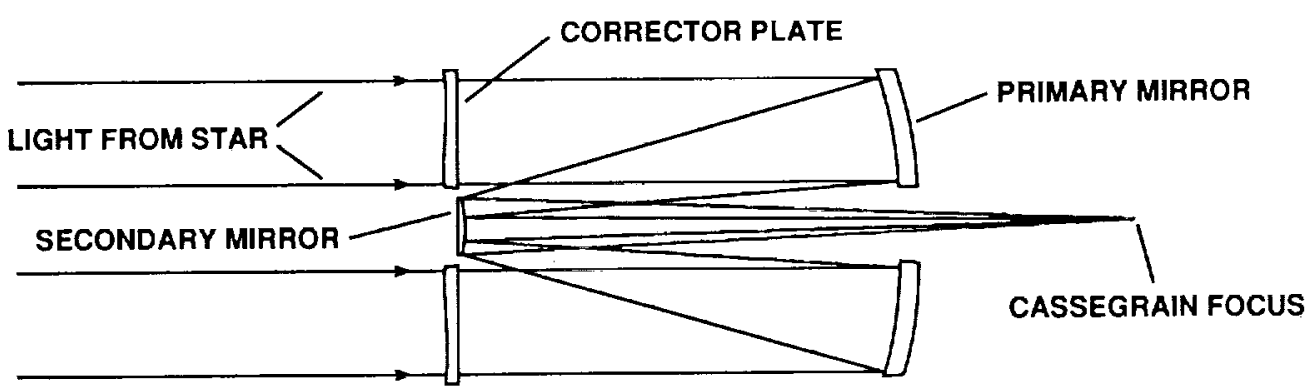

Figure 1. - Components of a Schmidt-Cassegrain telescope.

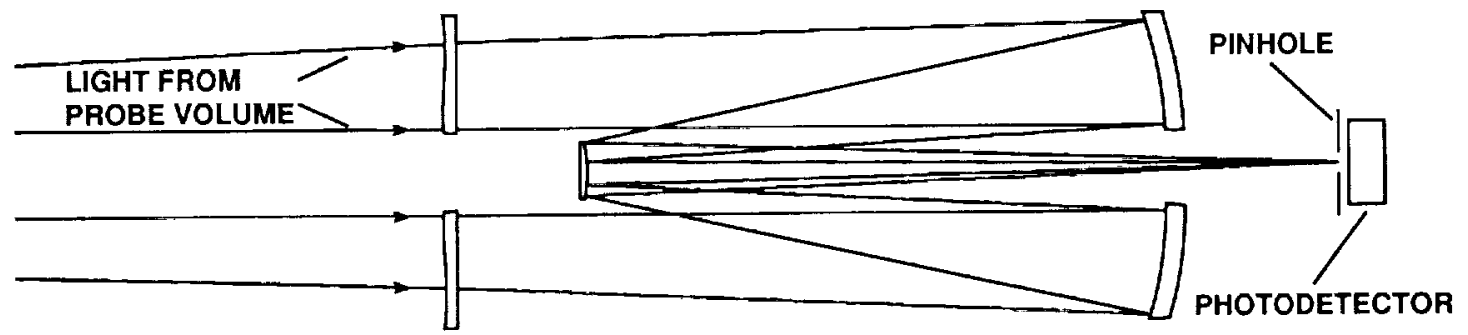

Figure 2. - Modified telescope for point focus collection.

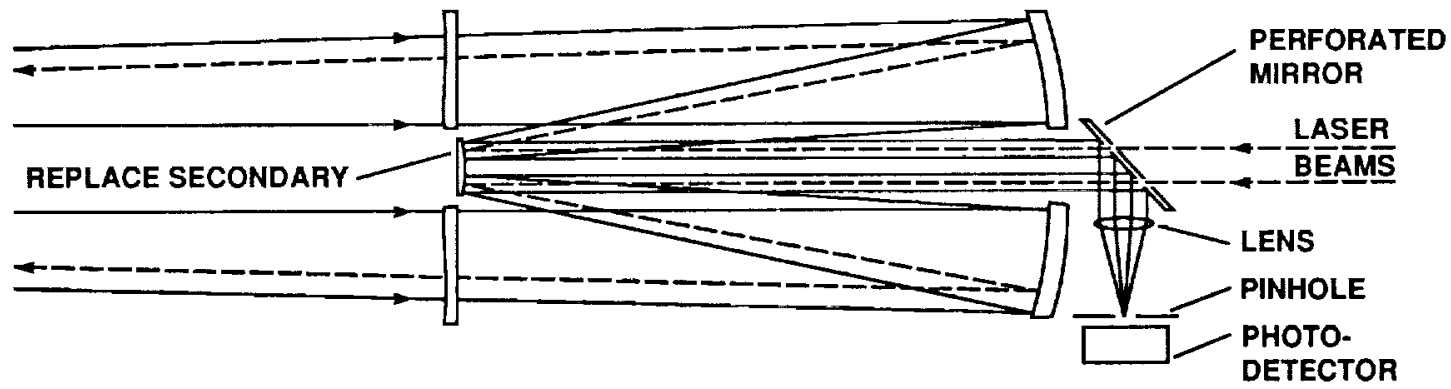

Figure 3. - Modified telescope for laser beam focusing and confocal backscatter collection. 


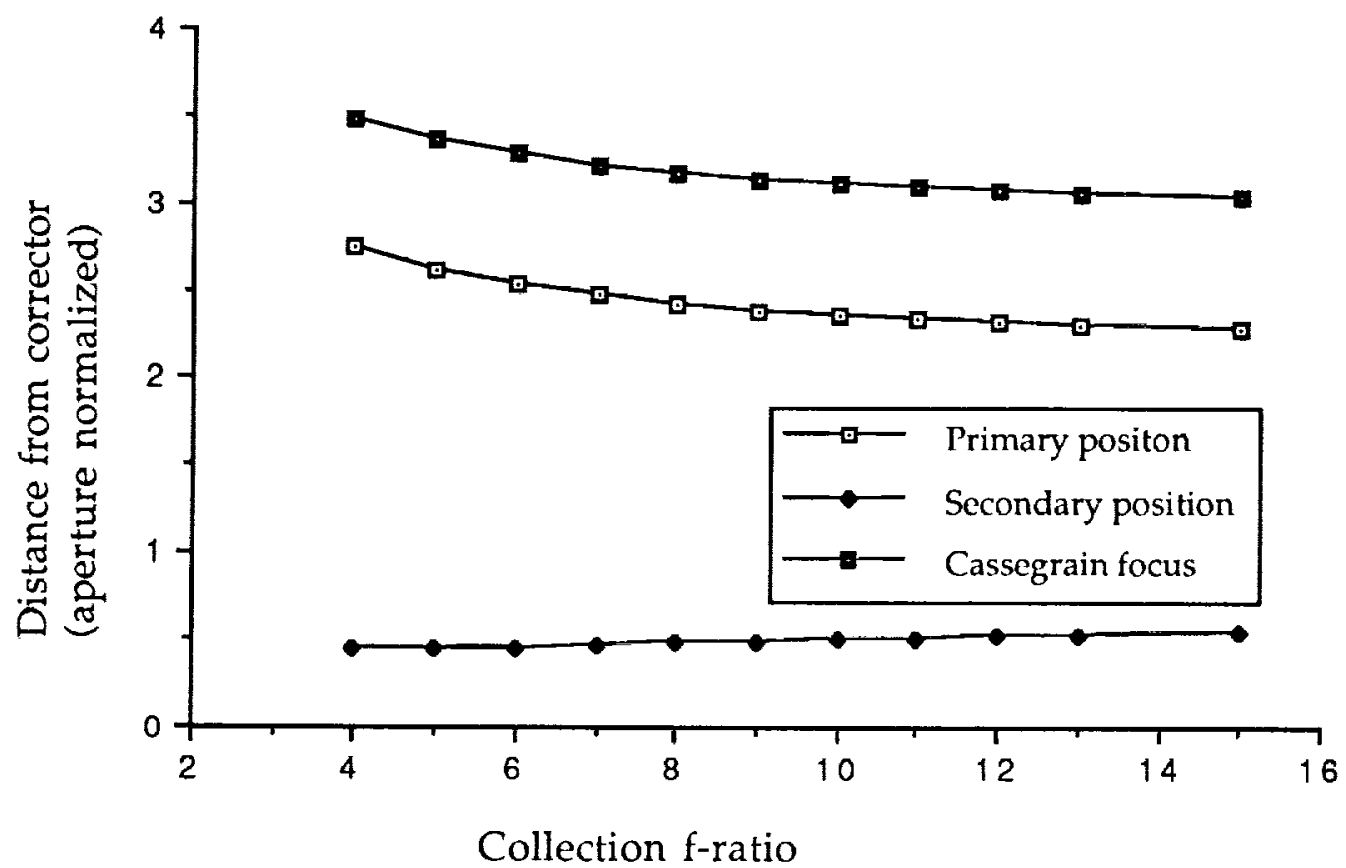

Figure 4 - Component locations as a function of collection range; repositioned primary and secondary mirror modification procedure for point focus collection.

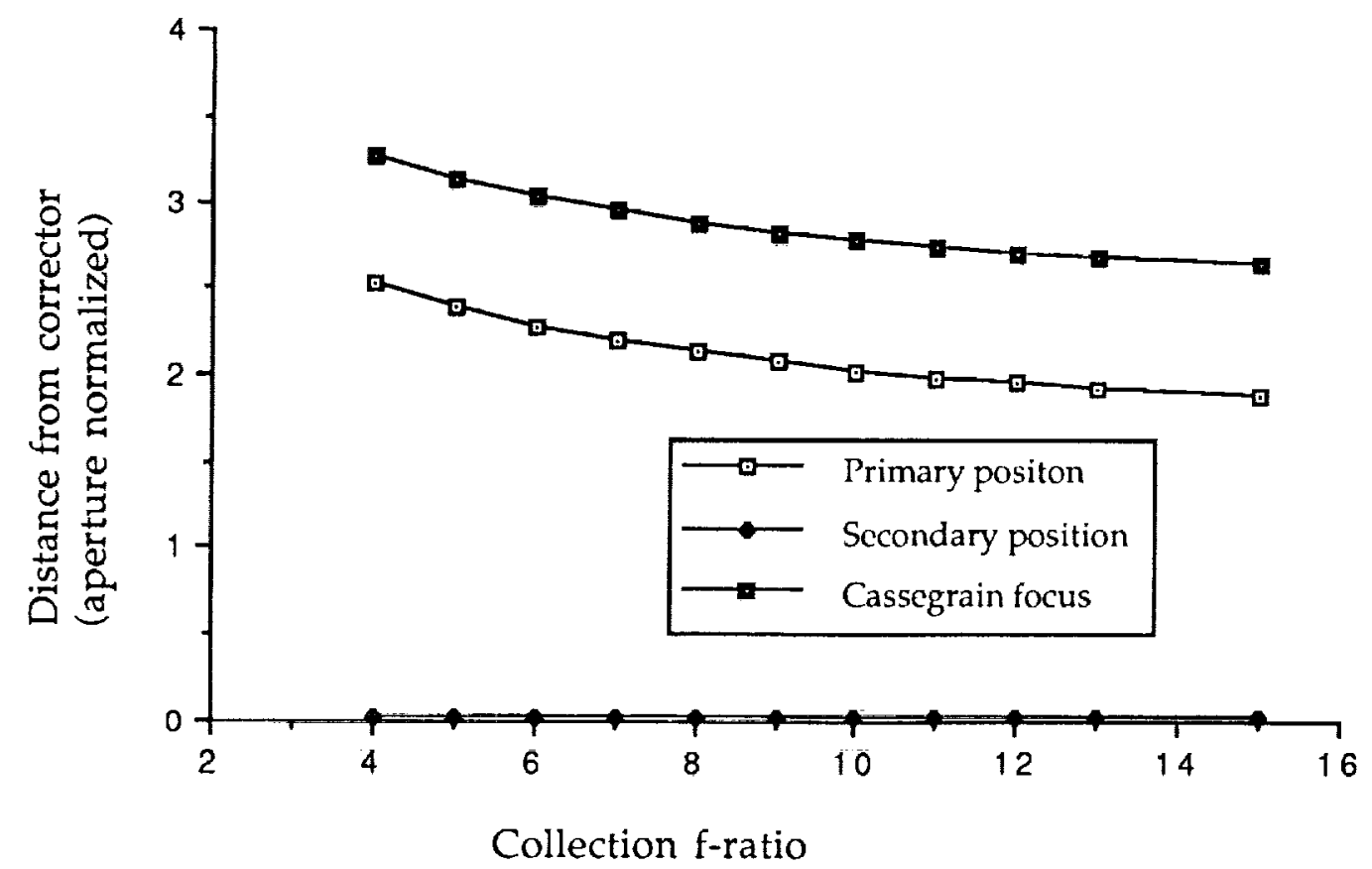

Figure 5. - Component locations as a function of collection range; repositioned primary and replacement secondary mirror modification procedure for confocal backscatter operation. 


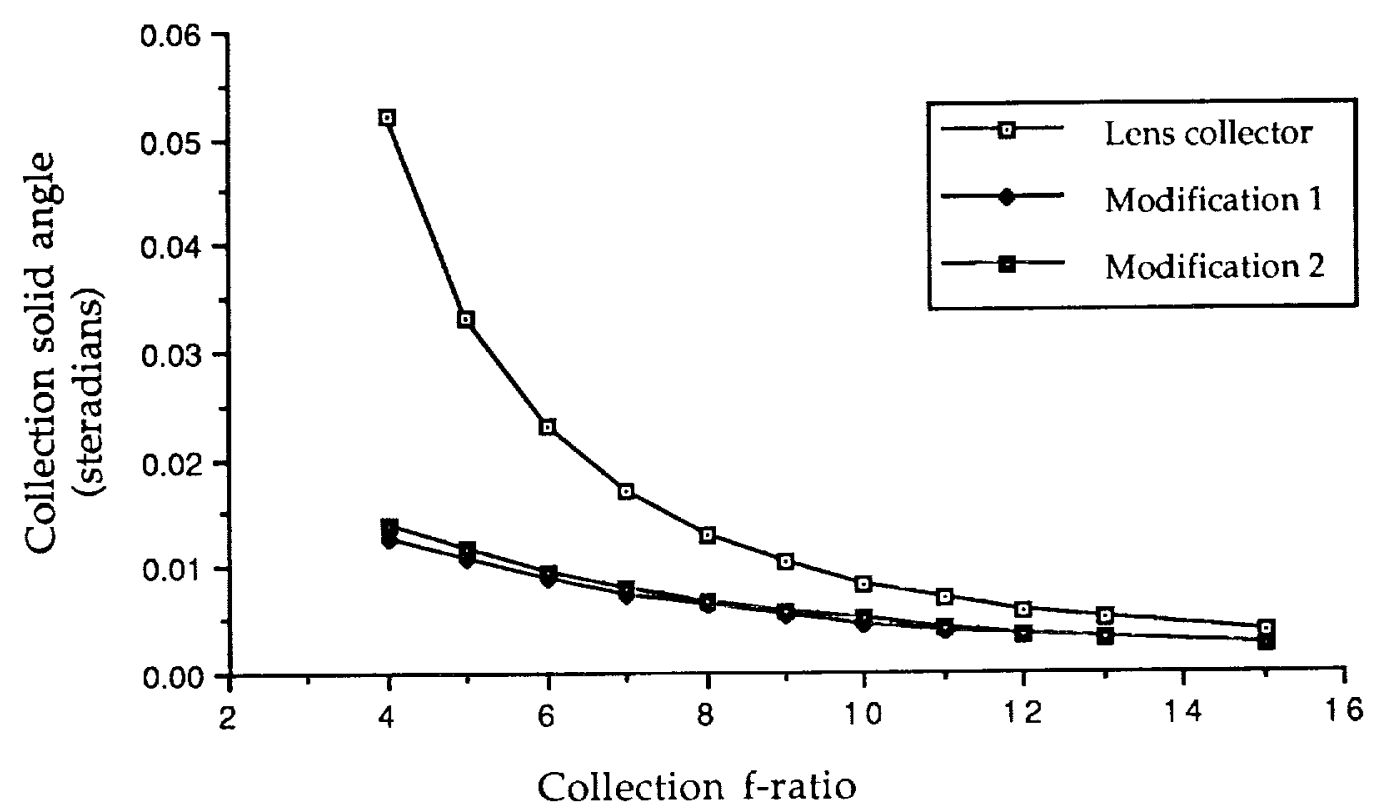

Figure 6. - Collection solid-angle comparisons for lens and telescope modifications.

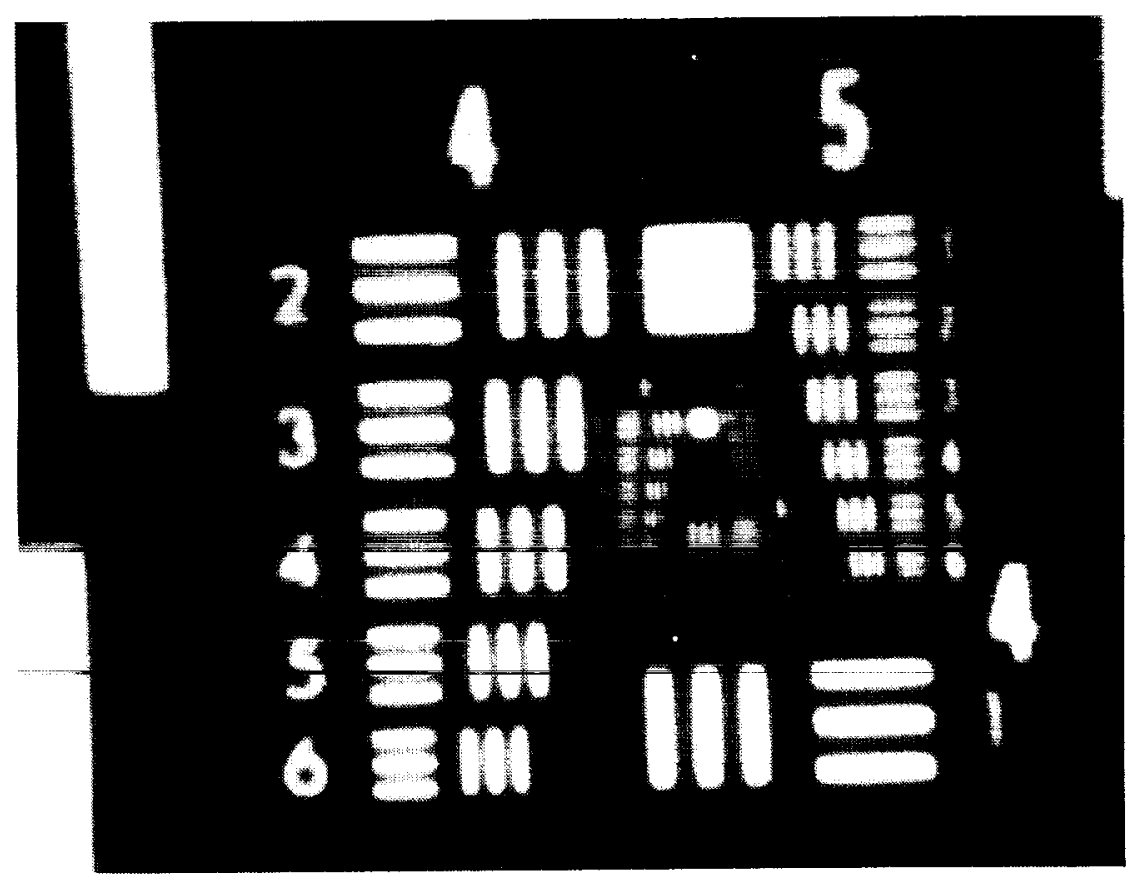

Figure 7. - USAF 1951 resolution target image. 


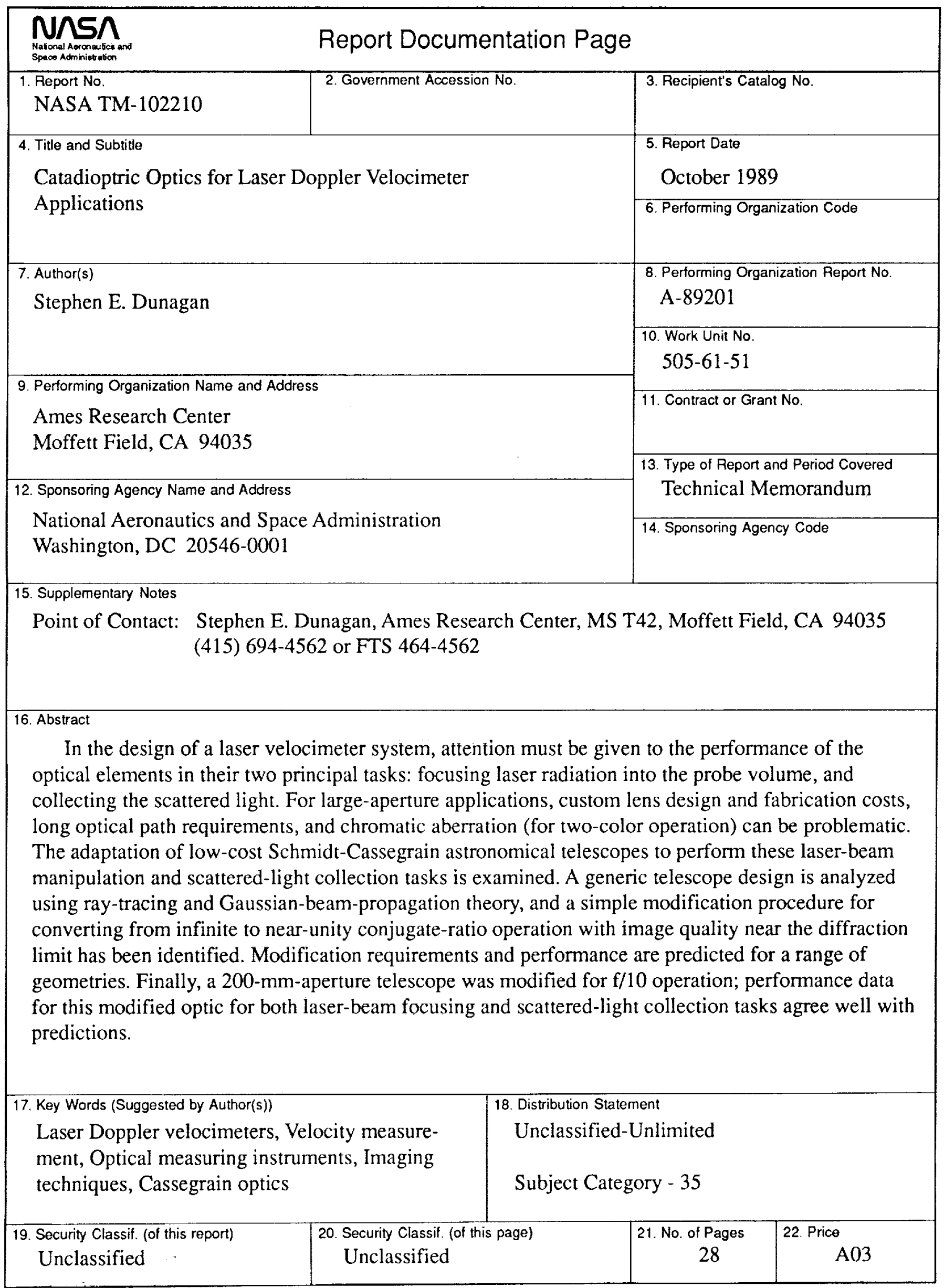

